Irish Math. Soc. Bulletin

Number 75, Summer 2015, 21-44

ISSN 0791-5578

\title{
INTEGRATION ISSUES IN PROBABILITY
}

\author{
PAT MULDOWNEY
}

\begin{abstract}
This essay explores the meaning of stochastic differential equations and stochastic integrals. It sets these subjects in a context of Riemann-Stieltjes integration. It is intended as a comment or supplement to [13].
\end{abstract}

\section{INTRODUCTION}

Famously, England and America are said to be divided by their common language. Similarly, mathematical analysts and probability theorists employ modes of expression which are superficially similar, but which may sometimes evoke different interpretations and connotations in each camp. This can be illustrated by the formula

$$
\int_{-\infty}^{x} \exp \left(-\frac{(y-\mu)^{2}}{2 \sigma^{2}}\right) \frac{d y}{\sigma \sqrt{2 \pi}} .
$$

To the analyst this expression may signify an improper indefinite integral, whereas the probabilist may see a cumulative normal distribution function. Aspects of the expression which are problematic or challenging to one may be trivially obvious to the other.

Another symptom is the probability/measure issue. In a kind of coup d'etat by mathematical analysis following the discoveries of A.N. Kolmogorov, the impression is sometimes given that the phenomenon of probability is now and forevermore to be understood in terms of the theory of measure.

But it is an overstatement to say that probability can be reduced to measure. Probability was a subject of interest long before modern measure theory existed, and there are aspects of random variation which are not amenable to explanation by the current methods of measure theory. On the other hand, an expert in probability is not,

2010 Mathematics Subject Classification. 65C30, 60H05.

Key words and phrases. Stochastic differential equation, Stochastic integral.

Received on 16-9-2014; revised 18-1-2015. 
by virtue of that alone, an expert in the theory of measure. Neither subject encompasses the other.

This essay seeks to tease out some distinctive features of these two mathematical disciplines in respect of topics such as Itô's formula and stochastic calculus. In particular, it aims to demonstrate how the modern theory of integration can shed light on some challenging aspects of random variation.

Mathematical analysis does not "own" probability theory. But, after all these years, it can still contribute to it!

\section{ITÔ'S FORMULA}

Itô's formula is an example of a stochastic differential equation:

$$
d Y_{s}=\frac{\partial f\left(X_{s}\right)}{\partial s} d s+\frac{1}{2} \frac{\partial^{2} f\left(X_{s}\right)}{\partial X_{s}^{2}} d s+\frac{\partial f\left(X_{s}\right)}{\partial X_{s}} d X_{s} .
$$

For $Y_{s}=f\left(X_{s}\right)$, this formula is an evocative, shorthand way of writing

$Y_{t}-Y_{0}=\int_{T} d Y_{s}=\int_{T} \frac{\partial f\left(X_{s}\right)}{\partial s} d s+\frac{1}{2} \int_{T} \frac{\partial^{2} f\left(X_{s}\right)}{\partial X_{s}^{2}} d s+\int_{T} \frac{\partial f\left(X_{s}\right)}{\partial X_{s}} d X_{s}$.

If the various expressions in this equation represented ordinary numbers and functions, then the presence in the equation of various integration processes might incline us to call (2) an integral equation.

But, while the symbol " $f$ " in both equations is actually an ordinary deterministic function (such as the operation of taking the square of some operand), the symbols $X$ and $Y$ do not represent "ordinary" functions or definite numbers. Instead, they are "random variables", that is, quantities which are indefinite or unknown, to the extent that they can be predicted only within some margin of error.

The presence of "=" in the equation indicates that it is an exact statement about actual quantities. Itô's formula can be best regarded as an exact statement about margins of error in estimates of uncertain quantities or measurements.

In other words, it deals with probability distributions of unpredictable quantities which are obtained by means of various operations in the formula, such as the integration operation. So Itô's formula can be regarded as a kind of integral equation in which the integrals are the type known as stochastic integrals. 
What is a stochastic integral? What is the meaning of Itô's formula? These questions are not trivial. They can be answered in a loose or intuitive manner, but deeper and more exact understanding can be challenging for non-specialists. And since they are a fundamental part of many important practical subjects, such as finance and communications, an understanding of them which is merely loose or hazy can be a serious barrier to competent practice in such subjects.

This essay seeks to outline an introduction to stochastic integrals which is less difficult than the standard textbook treatment of this subject. It uses Henstock's non-absolute integration instead of Lebesgue integration. It explores, compares, and contrasts these two methods of integration, with a view to assessing their role in stochastic integrals.

\section{RANDOM VARIABLES}

Broadly speaking - at the risk of haziness and looseness! - a random variable is a mathematical representation of a measurement (an experiment, trial, or observation) of some uncertain or unpredictable occurrence or value. For instance, the random variable $Z$ could represent a single throw of a die, so $Z$ represents possible outcomes $\{z=1, \ldots, z=6\}$ with probabilities $\left\{\frac{1}{6}, \ldots, \frac{1}{6}\right\}$. Or it could represent measurement of a standard normal variable whose possible values are the real numbers $z \in \mathbf{R}$, with standard normal probability distribution $\mathbf{N}(0,1)$.

Suppose the throw of the die yields a payoff or outcome $y=f(z)$ obtained by the following deterministic calculation:

$$
y=\left\{\begin{aligned}
-1 & \text { if } z=1 \\
+1 & \text { if } z=6 \\
0 & \text { otherwise }
\end{aligned}\right.
$$

This particular experiment or game depends on (is contingent on) the outcome of the experiment $Z$, and can be denoted by $Y=f(Z)$. Where $Z$ has six possible outcomes, with a uniform probability distribution, $Y$ has three possible outcomes whose probability distribution can easily be deduced by means of the deterministic calculation 
$f$. The probability distribution 11 of $Y$ is $y=-1$ with probability $\frac{1}{6}$, $y=+1$ with probability $\frac{1}{6}$, and $y=0$ with probability $\frac{2}{3}$.

We can easily invent such contingent random variables or gambling games using more than one throw of the die, and with payoff $Y$ dependent on some calculation based on the joint outcome of the successive throws.

This intuitive formulation is compatible with the formal and rigorous conception of a random variable as a $P$-measurable function whose domain is a $P$-measurable sample space $\Omega$. This twentieth century injection of mathematical rigor by A.N. Kolmogorov and others brought about a great extension of the depth and scope of the theory of probability and random variation, including the development of many new spheres of application of the theory.

These applications often involve stochastic processes. Suppose $T$ is some set of indexing elements $\{s\}$. For instance, $T$ could be an interval of real numbers $[a, b]$. A stochastic process $Y=Y_{T}$ is a family $Y=(Y(s))_{s \in T}$, for which each element $Y(s)=Y_{s}$ is a random variable. A sample path $(y(s))_{s \in T}$ of the process $Y=Y_{T}$ can be thought of as a function $y: T \mapsto \mathbf{R}$ in which, for each $s, y(s)$ (or $y_{s}$ ) is a possible outcome of the random variable (measurement, experiment, trial) $Y(s)$.

\section{Stochastic Integrals}

Take $T=[0, t]$. Equation (2) above appears to be the result of applying an integration operation $\int_{T}$ to the equation (1). If this is the case, and if this step is justified, then comparison of (1) and (2) implies (without delving into their actual meaning) that

$$
\int_{T} d Y_{s}=\int_{0}^{t} d Y_{s}=Y_{t}-Y_{0} ; \text { or } \int_{T} d Y(s)=\int_{0}^{t} d Y(s)=Y(t)-Y(0) .
$$

\footnotetext{
${ }^{1}$ The probability distribution ("margin of error") carries the essential information specifying the character of the random variable or experiment. It is often convenient to include other "potential" values or outcomes which are not actually possible or "potential". For instance, in the die-throwing experiment we can declare that every real number is a potential outcome. In that case we assign probability zero to the impossible outcomes. This does not change the random variable or its probability distribution in any essential way that affects its mathematical meaning
} 
On the face of it, a clear and precise understanding of this simplest of all possible stochastic integrals would seem to be the sine qua non of this subject. Expressed as a stochastic differential equation, it is the tautology $d Y_{s}=d Y_{s}$. Whatever (3) actually means, it seems consistent enough with more familiar forms of integration of the Stieltjes kind, in the somewhat loose and uncritical sense that the integral (or sum) of increments $d Y$ gives an overall increment.

Advancing a little bit further, take a deterministic function $f$, and consider $\int_{T} f\left(Y_{s}\right) d Y_{s}$ (or $\int_{T} f(Y(s)) d Y(s)$ ), which is a more general version of $\int_{T} d Y_{s}$. If $y$ is a sample path of the process $Y$, the expression

$$
\int_{T} f(y(s)) d y(s) \quad \text { or } \quad \int_{T} f\left(y_{s}\right) d y_{s}
$$

is a Stieltjes-type integral, which, if it exists, may be thought of as some limit of Riemann sums

$$
\sum f(y(s)) \Delta y(s) \text { or } \sum f\left(y\left(s_{j}\right)\right)\left(y\left(t_{j}\right)-y\left(t_{j-1}\right)\right)
$$

where the finite set of points $t_{j}$ form a partition of the interval $T=[0, t]$, with $t_{j-1} \leq s_{j} \leq t_{j}$ for each $j$.

From the point of view of basic mathematical analysis, unlike (3) which is about "margins of error" in probabilistic measurement, there is nothing problematic about (4) — this Riemann-Stieltjes-type integral may or may not exist for particular functions $y$ and $f$, but it is a fairly familiar subject for anyone who has studied basic Riemann-type integration.

In the Riemann sums for (4), some applications require that $s_{j}=$ $t_{j-1}$ for each $j$. Cauchy's approach to the theory of integration used approximating sums with $s_{j}=t_{j-1}$ or $s_{j}=t_{j}$, so such sums can be called Cauchy sums rather than Riemann sums. In any event, there are various ways, including the Lebesgue method, in which we can seek to define an integral $\int_{T} f(y(s)) d y(s)$ for sample paths $y_{T}=(y(s))_{s \in T}$ of a stochastic process $Y=Y_{T}$.

Suppose a Stieltjes-type integral of $f(y(s))$ is calculated with respect to the increments $y(I):=y\left(t_{j}\right)-y\left(t_{j-1}\right)$ of the function $y_{T}$. For instance, if $f$ is a function taking some fixed, real, constant value such as 1 , then a "naive" Riemann sum calculation on the domain $T=[0, t]$, with $t_{0}=0$ and $t_{n}=1$ gives

$$
\sum f(y(s)) y(I)=\sum_{j=1}^{n} y(I)=
$$


$\left(\left(y\left(t_{1}\right)-y(0)\right)+\left(\left(y\left(t_{2}\right)-y\left(t_{1}\right)\right)+\cdots+\left(\left(y(1)-y\left(t_{n-1}\right)\right)=y(1)-y(0)\right.\right.\right.$

for every sample outcome $y_{T}$ of the process $Y_{T}$. So it is reasonablein some "naive" way - to claim that, for this particular function $f$, the Riemann-Stieltjes integral exists for all outcomes $y_{T}$ :

$$
\int_{T} f\left(y_{s}\right) d y_{s}=\int_{0}^{t} d y(s)=y(t)-y(0) .
$$

One might then be tempted ${ }^{2}$ to apply such an argument to step functions $f$, and perhaps to try to extend it to some class of continuous functions $f$, especially if we are only concerned with sample paths $y_{T}$ which are continuous.

But the key point here is that, given a stochastic process $Y=Y_{T}$, and given certain deterministic functions $f$, real values

$\int_{T} f(y(s)) d y(s)$ can be obtained for each sample path $y=y_{T}$ by means of a recognizable Stieltjes integration procedure.

Can this class of real numbers or outcomes be related somehow to some identifiable random variable $Z$ which possesses some identifiable probability distribution (or "margin of error" estimates)?

If so, then $Z$ might reasonably be considered to be the random variable obtained by integrating, in some Stieltjes fashion, the random variable $f\left(Y_{s}\right)$ with respect to the increments $Y(I)=Y\left(t_{j}\right)-$ $Y\left(t_{j-1}\right)$ of the stochastic process $Y_{T}$.

In other words, $Z$ is the stochastic integral $\int_{T} f\left(Y_{s}\right) d Y_{s}$.

To justify the latter step, a probability distribution (or "margin of error" data) for $Z$ must be determined. But, in the case of the constant function $f$ given above $\left(f\left(y_{s}\right)=1\right)$, this is straightforward. Because, with $f\left(y_{s}\right)=1$ for all outcomes $y_{s}$ in all sample paths (or joint outcomes) $y_{T}$, the distribution function obtained for the Riemann sum values $\sum f\left(y_{s}\right) y(I)$ is simply the known distribution function of the outcomes $y(t)-y(0)$ of the random variable $Y(t)-$ $Y(0)$.

This distribution is the same for all partitions of $T=[0, t]$. So it is reasonable to take it to be the distribution function of the stochastic integral $Z=\int_{T} f\left(Y_{s}\right) d Y_{s}$. For constant $f$ this seems to provide meaning and rationale for (4).

\footnotetext{
${ }^{2} \mathrm{~A}$ warning against this temptation is provided in Example 8.3 below.
} 
What this amounts to is a naive or intuitive interpretation of stochastic integration which seems to hold for some elementary functions $f$. This approach can be pursued further to give a straightforward interpretation - indeed, a "proof" - of Itô's formula, at least for the unchallenging functions $f$ mentioned above.

But what of the standard or rigorous theory of stochastic integration?

\section{Standard THEORY OF STOCHASTIC INTEGRATION}

Unfortunately, this theory cannot accommodate the naive or intuitive construction of the simple stochastic integrals described in the preceding section. Broadly speaking, the elementary Riemann sum type of calculation is not adequate for the kinds of analysis needed in this subject. It is not possible, for instance, to apply a monotone convergence theorem, or a dominated convergence theorem, to simple Riemann and Riemann-Stieltjes integrals. Historically, these kinds of analysis and proof have been supplied by Lebesgue-type integrals which, while requiring a measure function as integrator, cannot be simply defined by means of the usual arrangement $t^{3}$ of Riemann sums.

And this is where the difficulty is located. Suppose, for instance, that the stochastic process $Y_{T}$ that we are dealing with is a standard Brownian motion. In that case any sample path $y_{T}$ is, on the one hand, almost surely continuous - which is "nice"; but, on the other hand, it is almost surely not of bounded variation in every interval $J$ of the domain $T=[0,1]$. And the latter is "nasty".

This turns out to be very troublesome if we wish to construct a Lebesgue-Stieltjes integral using the increments $y(I)=y\left(t_{j}\right)-$ $y\left(t_{j-1}\right)$ of a sample path which is continuous but not of bounded variation in any interval.

The problem is that, in order to construct a Lebesgue-Stieltjes measure from the increments $y(I)$, we must separate the non-negative increments $y_{+}(I)$ from the negative-valued increments $y_{-}(I)$,

$$
y(I)=y_{+}(I)-\left|y_{-}(I)\right|,
$$

and try to construct a non-negative measure from each of the components. But, because $y$ is not of bounded variation, the construction

\footnotetext{
${ }^{3}$ But Section 8 shows that Lebesgue integrals are essentially Riemann-Stieltjes integrals.
} 
for each component diverges to infinity on every interval $J$. Thus the standard theory of stochastic integration encounters a significant difficulty at the very first step (4).

To summarize:

- In the standard Itô or Lebesgue integral approach, the most basic calculation of the integral of a constant function $f\left(Y_{T}\right)$, with respect to the increments $d Y$ of a Brownian process, fails because the Lebesgue-Stieltjes measure does not exist.

- On the other hand, if Riemann sums of the increments of the process $Y_{T}$ are used, then, by cancellation, a finite result is obtained for each Riemann sum - a result which agrees with what is intuitively expected.

In the standard Lebesgue (or Itô) theory of stochastic integrationin [16] for instance - this problem is evaded by postulating a finite measure $\mu_{y}(J)$ for each sample path, and then constructing a weak form of integral which, in the case of Brownian motion, is based on certain helpful properties of this process.

The trouble with this approach is that it produces a quite difficult theory which does not lend itself to the natural, intuitive interpretation described above.

However, elementary Riemann-sum-based integration is not generally considered to have the analytical power possessed by Lebesguestyle integration. And a great deal of analytical power is required in the theory of stochastic processes. So at first sight it seems that we are stuck with the standard theory of stochastic integration, along with all its baggage of subtlety and complication.

But this is not really the case. The good news is that is actually possible to formulate the theory of stochastic integrals using Riemann sums instead of the measures of Lebesgue theory.

\section{INTEGRATION OF FUNCTIONS}

To see this, it is first necessary to review the various kinds of integration which are available to us.

First consider the basic Riemann integral, $\int_{a}^{b} f(s) d s$, of a realvalued, bounded, continuous function $f(s)$ on an interval $[a, b]$. Let $\mathcal{P}$ be a partition of $[a, b]$;

$$
\mathcal{P}: \quad a=t_{0}<t_{1}<t_{2}<\cdots<t_{n}=b,
$$


for any choice of positive integer $n$ and any choice of $t_{j}, 1 \leq j<n$. For any $u<v$ and any interval $I$ with end-points $u$ and $v$, write $|I|=v-u$. Denoting intervals $\left.] t_{j-1}, t_{j}\right]$ by $I_{j}$ let

$$
U_{\mathcal{P}}=\sum_{j=1}^{n} P_{j}\left|I_{j}\right|, \quad L_{\mathcal{P}}=\sum_{j=1}^{n} p_{j}\left|I_{j}\right|
$$

where

$$
P_{j}=\sup \left\{f(s): s \in I_{j}\right\}, \quad p_{j}=\inf \left\{f(s): s \in I_{j}\right\} .
$$

Definition 6.1. Define the upper Riemann integral of $f$ by

$$
U:=\inf \left\{L_{\mathcal{P}}: \text { all partitions } \mathcal{P} \text { of }[a, b]\right\},
$$

and the lower Riemann integral of $f$ by

$$
L:=\sup \left\{l_{\mathcal{P}}: \text { all partitions } \mathcal{P} \text { of }[a, b]\right\} .
$$

Then $U_{\mathcal{P}} \geq L_{\mathcal{P}}$ for all $\mathcal{P}$, and if $U=L$ we say that $f$ is Riemann integrable, with

$$
\int_{a}^{b} f(s) d s:=U=L .
$$

Write the partition $\mathcal{P}$ as $\{I\}$ where each $I$ has the form $\left.\left.I_{j}=\right] t_{j-1}, t_{j}\right]$, with $\left|I_{j}\right|=t_{j}-t_{j-1}$, and Riemann sum

$$
(\mathcal{P}) \sum f(s)|I|=\sum_{j=1}^{n} f\left(s_{j}\right)\left|I_{j}\right| .
$$

Suppose $g(s)$ is a real-valued, monotone increasing function of $s \in$ $[a, b]$, so $g(s) \geq g\left(s^{\prime}\right)$ for $s>s^{\prime}$. For any interval $I$ with end-points $u$ and $v(u<v)$, define the increment or interval function $g(I)$ to be $g(v)-g(u)$.

Definition 6.2. If $|I|$ and $\left|I_{j}\right|$ are replaced by $g(I)$ and $g\left(I_{j}\right)$ in Definition 6.1 of the Riemann integral, then the resulting integral is called the Riemann-Stieltjes integral of $f$ with respect to $g, \int_{a}^{b} f d g$ or $\int_{a}^{b} f(s) d g(s)$.

In fact if we start with the latter definition the Riemann integral is a special case of it, obtained by taking the point function $g$ to be the identity function $g(s)=s$.

If $g(s)$ has bounded variation it can be expressed as the difference of two monotone increasing, non-negative point functions,

$$
g(s)=g_{+}(s)-\left(-g_{-}(s)\right)
$$


and the Riemann-Stieltjes integral of $f$ with respect to $g$ can then be defined as the difference of the Riemann-Stieltjes integrals of $f$ with respect to $g_{+}$and $-g_{-}$, respectively.

The following result is well known: if real-valued, bounded $f$ is continuous and if real-valued $g$ has bounded variation then $\int_{a}^{b} f d g$ exists.

As suggested earlier, the Lebesgue integral of a real-valued point function $k$ with respect to a measure $\mu$ can be viewed, essentially, as a Riemann-Stieltjes integral in which the point-integrand $k(\omega)$ satisfies the condition of measurability. To explain this statement further, consider a measure space $(\Omega, \mathcal{A}, \mu)$ with non-negative measure $\mu$ on a sigma-algebra $\mathcal{A}$ of $\mu$-measurable subsets of the arbitrary measurable space $\Omega$. Thus, if $\mu(\Omega)=1$, the measure space is a probability space. Suppose the point-integrand $k$ is a bounded real-valued $\mu$-measurable function on the domain $\Omega$. Then there exist real numbers $c$ and $d$ for which

$$
c \leq k(\omega) \leq d \quad \text { for all } \omega \in \Omega .
$$

Also, for each sub-interval $J$ of $[c, d]$, measurability of $k$ implies $\mu\left(k^{-1}(J)\right)$ is defined. The basic definition of the Lebesgue integral of $k$ with respect to $\mu$ on $\Omega$ is as follows.

Definition 6.3. Let $\left.\left.\mathcal{Q}=\left\{J_{j}\right\}=\{] v_{j-1}, v_{j}\right]\right\}$ be a partition of $[c, d]$,

$$
\mathcal{Q}: \quad c=v_{0}<v_{1}<v_{2}<\cdots<v_{n}=d,
$$

and let

$$
L_{\mathcal{Q}}=\sum_{j=1}^{n} v_{j-1} \mu\left(k^{-1}\left(J_{j}\right)\right), \quad U_{\mathcal{Q}}=\sum_{j=1}^{n} v_{j} \mu\left(k^{-1}\left(J_{j}\right)\right) .
$$

Let $L:=\sup \left\{L_{\mathcal{Q}}: \mathcal{Q}\right\}, U:=\inf \left\{U_{\mathcal{Q}}: \mathcal{Q}\right\}$, the supremum and infimum being taken over all partitions $\mathcal{Q}$ of $[c, d]$. If $L=U$, then their common value is the Lebesgue integral $\int_{\Omega} k(\omega) d \mu$.

An advantage of Lebesgue integration over Riemann integration is that the former has theorems, such as the dominated and monotone convergence theorems which, under certain condition, make it possible for instance to change the order of integration and differentiation. Also, Fubini's and Tonelli's theorems allow exchange of order of multiple integrals.

What makes "good" properties such as these possible is measurability of the integrand $k$. But the Lebesgue integral itself is, by 
definition, a Riemann-Stieltjes-type integral. To see this, for each $u \in[c, d]$ define the monotone increasing function

$$
g(u)=\mu\left(k^{-1}([c, u])\right),
$$

and take the point function $h(u)$ to be the identity function $h(u)=$ $u$. Then the construction 4 in Definition 6.3 shows that

$$
\int_{\Omega} k(\omega) d \mu=\int_{c}^{d} h(u) d g(u),=\int_{c}^{d} u d g .
$$

In other words, when combined with the measurability property of the point-integrand, this particular Riemann-Stieltjes construction gives the "good" properties required in the integration of functions.

\section{RiEMANn DEFINITION}

But in fact a Riemann construction can give these "good" properties without postulating measurability in the definition! of the integral. To see this, we start again by considering a more general and more flexible definition of basic Riemann and Riemann-Stieltjes integration which generalizes the construction of these integrals as given above in Definitions 6.1 and 6.2.

The proposed, more general, definition of the Riemann-Stieltjes integral is applicable to real- or complex-valued functions $f$ (bounded or not); and to real- or complex-valued functions $g$, with or without bounded variation.

Definition 7.1. The function $f$ is Riemann-Stieltjes integrable with respect to $g$, with integral $\alpha$, if, given $\varepsilon>0$, there exists a constant $\delta>0$ such that, for every partition $\mathcal{P}=\{I\}$ of $[a, b]$ satisfying $|I|<\delta$ for each $I \in \mathcal{P}$, the corresponding Riemann sum satisfies

$$
\left|\alpha-(\mathcal{P}) \sum f(s) g(I)\right|<\varepsilon,
$$

so $\alpha=\int_{a}^{b} f d g$.

\footnotetext{
${ }^{4}$ The integral of a point function $h(u)$ with respect to a point function $g(u)$ can be addressed either as a Riemann-Stieltjes construction or as a Lebesgue-Stieltjes construction. When $h(u)=u$ and $g(u)=\mu\left(k^{-1}([c, u])\right)$ the former approach gives the Lebesgue integral $\int_{\Omega} k(\omega) d \mu$. On the other hand, if the Lebesgue-Stieltjes construction is attempted with $h(u)=u$ and $g(u)=\mu\left(k^{-1}([c, u])\right)$, we simply replicate the Riemann-Stieltjes construction of the Lebesgue integral $\int_{\Omega} k(\omega) d \mu$, and nothing new emerges.

${ }^{5}$ And if measurability is redundant in the definition, then so is the measure space structure.
} 
If $g$ is the identity function $g(s)=s$ then Definition 7.1 reduces to the ordinary Riemann integral of $f, \int_{a}^{b} f(s) d s$.

Definition 7.1 does not embody conditions which ensure the existence of the integral. Such integrability conditions are not postulated but are deduced, in the form of theorems, from the definition of the integral.

Thus, if the function properties specified, respectively, in Definitions 6.1, 6.2, and 6.3 above are assumed, the integrability in each case follows from Definition 7.1; and Definitions 6.1, 6.2, and 6.3 become theorems of Riemann, Riemann-Stieltjes, and Lebesgue integration, respectively.

Definition 6.3 can now be expressed in terms of Definition 7.1, using the formulations (5) and (6), and assuming measurability of the integrand $f$ with respect to measure space $(\Omega, \mathcal{A}, \mu)$.

Definition 7.2. The function $f$ is Lebesgue integrable with respect to measure $\mu$, with integral $\int_{\Omega} f(\omega) d \mu=\alpha$, if, given $\varepsilon>0$, there exists a constant $\delta>0$ such that, for every partition $\mathcal{Q}=\{J\}$ of $[c, d]$ satisfying $|J|<\delta$ for each $J \in \mathcal{Q}$, the corresponding Riemann sum satisfies

$$
\left|\alpha-(\mathcal{Q}) \sum h(u) g(J)\right|<\varepsilon,
$$

where $h(u)=u$ is the identity function on $[c, d]$; so $\alpha=\int_{c}^{d} h(u) d g(u)$ $=\int_{c}^{d} u d g$.

Thus, by definition, the Lebesgue integral $\int_{\Omega} f(\omega) d \mu$, with domain $\Omega$, is the Riemann-Stieltjes integral $\int_{c}^{d} u d g$, with domain $[c, d]$.

The following result is an obvious consequence of Definition 7.1. If $f$ has constant value $\beta$ and if $g$ is an arbitrary real- or complexvalued function, then $\int_{a}^{b} f d g$ exists and equals $\beta(g(b)-g(a))$. This follows directly from Definition 7.1 since, for every partition $\mathcal{P}$ of $[a, b]$, cancellation of terms gives

$$
(\mathcal{P}) \sum f(s) g(I)=\beta \sum_{j=1}^{n}\left(g\left(t_{j}\right)-g\left(t_{j-1}\right)\right)=\beta(g(b)-g(a)) .
$$

This result does not in general hold for Lebesgue-Stieltjes integration, as the latter requires that $g(s)$ be resolved into its negative and non-negative components, $g(s)=g_{+}(s)-\left(-g_{-}(s)\right)$, and convergence may fail when the integral is calculated with respect to each of these components separately. 
Example 8.3 below shows that, though constant functions $f$ are Riemann-Stieltjes integrable with respect to any integrator function $g$, this does not necessarily extend to step functions $f$.

Definition 7.1 of the Riemann or Riemann-Stieltjes integral does not postulate any boundedness, continuity, measurability or other conditions for the integrand $f$. But, as already stated, in the absence of integrand measurability and the construction in Definition 6.3 , this method of integration does not deliver good versions of monotone and dominated convergence theorems, or Fubini's theorem.

\section{8. -COMPlete INTEGRATiON}

Developments in the subject since the 1950's - developments which were originated independently by R. Henstock and J. Kurzweilhave made good this deficit in the basic Riemann and RiemannStieltjes construction. In this new development of the subject, Definition 7.1 of the Riemann-Stieltjes integral is amended as follows.

Definition 8.1. A function $f$ is Stieltjes-complete integrable with respect to a function $g$, with integral $\alpha$ if, given $\varepsilon>0$, there exists a function $\delta(s)>0$ such that

$$
\left|\alpha-(\mathcal{P}) \sum f(s) g(I)\right|<\varepsilon
$$

for every partition $\mathcal{P}$ such that, in each term $f(s) g(I)$ of the Riemann sum, we have $s-\delta(s)<u \leq s \leq v<s+\delta(s)$, where $u$ and $v$ are the end-points of the partitioning interval $I$.

In other words, where $|I|$ is less than a constant $\delta$ in the basic Riemann-Stieltjes definition, we have $|I|<\delta(s)$ in the new definition. Write $\alpha=\int_{[a, b]} f(s) g(I)$, or $\int_{[a, b]} f d g$, for the Stieltjescomplete integral whenever it exists.

Again, if the integrator function $g$ is the identity function $g(s)=s$, the resulting integral (corresponding to the basic Riemann integral), is the Riemann-complete integral of $f$, written $\alpha=\int_{[a, b]} f(s)|I|$, or $\int_{[a, b]} f(s) d s$. The latter is also known as the Henstock integral, the Kurzweil integral, the Henstock-Kurzweil, the generalized Riemann integral, or the gauge integral since in this context the function $\delta(s)>0$ is called a gauge.

It is obvious that every Riemann (Riemann-Stieltjes) integrable integrand is also Riemann-complete (Stieltjes-complete) integrable, 
as the gauge function $\delta(s)>0$ of Definition 7.1 can be taken to be the constant $\delta>0$ of Definition 6.1 and Definition 6.2.

This argument indicates a Lebesgue-complete extension of the Lebesgue integral, by replacing the constant $\delta>0$ of Definition 7.2 with a variable gauge $\delta(u)>0$ :

Definition 8.2. Let $h(u)=u$ be the identity function on $[c, d]$. The function $f$ is Lebesgue-complete integrable with respect to measure $\mu$, with integral $\int_{\Omega} f d \mu=\alpha$, if, given $\varepsilon>0$, there exists a gauge $\delta(u)>0$ for $c \leq u \leq d$, such that

$$
\left|\alpha-(\mathcal{Q}) \sum h(u) g(J)\right|<\varepsilon,
$$

for every partition $\mathcal{Q}=\{J\}$ of $[c, d]$ satisfying

$$
u-\delta(u)<v_{j-1} \leq u \leq v_{j}<u+\delta(u)
$$

for each $\left.J=] v_{j-1}, v_{j}\right] \in \mathcal{Q}$.

In that case $\alpha=\int_{[c, d]} h(u) g(J)=\int_{[c, d]} u g(J)$, and the Lebesguecomplete integral is a special case of the Stieltjes-complete integrala special case in which a measure space structure exists and for which the integrand is measurable. So it is again clear that every Lebesgue integrable integrand is Lebesgue-complete integrable; since the former is, in effect, a Riemann-Stieltjes integral, the latter is a Stieltjescomplete integral, and every Riemann-Stieltjes integrable function is also Stieltjes-complete integrable. (No special notation has been introduced here to distinguish the Lebesgue integral $\int_{\Omega} f d \mu$ from its Lebesgue-complete counterpart.)

If the measurable domain $\Omega$ is a real interval such as $[a, b]$, then some ambiguity arises in the interpretation of the Lebesgue integral as an integral of the gauge, or generalized Riemann, kind. The reason for the ambiguity is as follows. Assuming the existence of the Lebesgue integral $\int_{\Omega} f(\omega) d \mu,=\int_{[a, b]} f(\omega) d \mu$, where $\omega$ now represents real numbers in the domain $[a, b]$, then we are assured of the existence of the Stieltjes and Stieltjes-complete (or Lebesgue-complete) integrals $\int_{c}^{d} u d g$ and $\int_{[c, d]} u g(J)$, respectively, with

$$
\int_{[a, b]} f(\omega) d \mu=\int_{c}^{d} u d g=\int_{[c, d]} u g(J),
$$

where the values $u=h(u)$ are elements of $[c, d]$ and $h$ is the identity function on $[c, d]$. 
But in this case, letting $\omega=s$ denote points of the domain $[a, b]$ and with $I$ denoting subintervals of $[a, b]$, the function $\mu(I)$ is defined on intervals $I$, and two different Stieltjes-type constructions are possible.

First, there is the Riemann-Stieltjes integral $\int_{c}^{d} u d g$ which defines the Lebesgue integral $\int_{\Omega} f(\omega) d \mu,=\int_{[a, b]} f(\omega) d \mu$. Secondly, there is the gauge integral $\int_{[a, b]} f(s) \mu(I)$ which has a Stieltjes-complete construction.

It is then meaningful to consider whether, with $f$ measurable, existence of the Lebesgue integral $\int_{[a, b]} f(\omega) d \mu$ implies existence of the Stieltjes-complete integral $\int_{[a, b]} f(s) \mu(I)$, and whether

$$
\int_{c}^{d} u d g=\int_{[a, b]} f(s) \mu(I)
$$

holds, ${ }^{6}$ the first of these integrals being the Lebesgue integral $\int_{[a, b]} f(\omega) d \mu$, which, by Definition 7.2 , is interpreted as the RiemannStieltjes integral $\int_{c}^{d} u d g$.

To see that these two integrals coincide, take $f$ to be a bounded, measurable function on $[a, b]$. This can be expressed as the difference of two non-negative, bounded, measurable functions $f_{+}$and $f_{-}$. Accordingly, and without loss of generality, take $f$ to be non-negative, bounded, measurable. Then the Lebesgue integrable function $f$ is the $\mu$-almost everywhere point-wise limit of a monotone increasing sequence of step functions $f_{j}$. With $\omega=s$, each step function $f_{j}$ is Lebesgue integrable, with Lebesgue integral $\int_{[a, b]} f_{j}(\omega) d \mu$; and each step function $f_{j}$ is Stieltjes-complete integrable, with Stieltjescomplete integral $\int_{[a, b]} f_{j}(s) \mu(I)$, and

$$
\int_{[a, b]} f_{j}(\omega) d \mu=\int_{[a, b]} f_{j}(s) \mu(I)
$$

for each $j$. (This statement is also true if "Lebesgue integral" and "Lebesgue integrability" are replaced by "Lebesgue-complete integral" and "Lebesgue-complete integrability".)

\footnotetext{
${ }^{6}$ There is a considerable literature on this question, which is usually answered as: "Every Lebesgue integrable function on an interval of the real numbers $\mathbf{R}$ is also Henstock-Kurzweil integrable." If the domain of the integrand is a measurable space $\Omega$ which is not a subset of $\mathbf{R}$ or $\mathbf{R}^{n}$, then the appropriate way to formulate the corresponding Henstock-Kurzweil (or -complete) integral is in the form $\int_{[c, d]} u g(J)$ described in Definition 8.2.
} 
By the monotone convergence theorem of Lebesgue integration (or, respectively, by the monotone convergence theorem of Lebesguecomplete integration),

$$
\int_{[a, b]} f_{j}(\omega) d \mu \rightarrow \int_{[a, b]} f(\omega) d \mu
$$

as $j \rightarrow \infty$. By the monotone convergence theorem of Stieltjescomplete integration, $f(s) \mu(I)$ is Stieltjes-complete integrable and

$$
\int_{[a, b]} f_{j}(s) \mu(I) \rightarrow \int_{[a, b]} f(s) \mu(I)
$$

as $j \rightarrow \infty$. Since corresponding integrals of the pair of sequences are equal, their limits are equal:

$$
\int_{[a, b]} f(\omega) d \mu=\int_{[a, b]} f(s) \mu(I) .
$$

This is the gist of a proof that existence of a Lebesgue integral (or of a Lebesgue integral) on a real domain implies existence of the corresponding Stieltjes-complete integral on the same domain, and equality of the two.

Thus the above argument can be applied to either the Lebesgue or the Lebesgue-complete integral on $\Omega=[a, b]$ in conjunction, respectively, with the corresponding Stieltjes-complete integral on the same domain. In effect, if the domain $\Omega$ is a subset of $\mathbf{R}$, and if $f$ is Lebesgue integrable or Lebesgue-complete integrable with respect to $\mu$, then $f(s) \mu(I)$ is also Stieltjes-complete integrable and the two integrals are equal.

The specific properties of the Lebesgue-complete integral have not been investigated.

As mentioned earlier, constant functions $f$ are Riemann-Stieltjes integrable, and hence Stieltjes-complete integrable, with respect to any integrator function $g$. But as the following counter-example shows, this does not necessarily extend to step functions $f$, or any other functions which are not constant.

Example 8.3. Dirichlet function: For $0 \leq s \leq 1$ let $D(s)$ be 1 if $s$ is rational, and 0 otherwise. For $I=] u, v]$ let $D(I)=D(v)-D(u)$. Let $D([0, v])=D(v)-D(0)$. The point function $D(s)$ is discontinuous everywhere, and has infinite variation on every interval $J \subseteq[0,1]$. If $f(s)$ is constant for $0 \leq s \leq 1$, then the RiemannStieltjes integral $\int_{0}^{1} f(s) d D$ exists and equals $D(1)-D(0)$; that is, 
$\int_{0}^{1} f(s) d D=0$. But if $f$ is not constant on $[0,1]$, then the Riemann-Stieltjes integral of $f$ with respect to $D$ does not exist. What about Stieltjes-complete integrability of $f(s) D(I)$ ? In fact, if $f$ is not constant on $[0,1]$, then the Stieltjes-complete integral of $f$ with respect to $D$ does not exist. This is proved in Theorem 1 of [6], and the proof is reproduced in Theorem 67 of [13]. Thus $f(s) D(I)$ is Riemann-Stieltjes integrable and Stieltjescomplete integrable on $[0,1]$ if and only if $f(s)$ is constant for $0 \leq s \leq 1$.

Historically this is the first published result (Theorem 1 of [6]) in the theory of -complete integration.

\section{9. -Complete Approach to Stochastic integrals}

Returning to stochastic integrals, the -complete method of integration allows us to construct Stieltjes-type Riemann sums for highly oscillatory expressions which include both positive and negative terms. Cancellation of terms can occur in the Riemann sum approximations, so the possibility of convergence is preserved by this construction.

The Lebesgue construction, on the other hand, requires integral convergence, separately and independently, of the positive and negative components of the integrand. The difficulty this presents is illustrated in the alternating or oscillating series $\sum_{j=1}^{\infty}(-1)^{j} j^{-1}$. If the positive and negative terms of the series are considered as two separate series then each of them diverges. But the series itself is conditionally (or non-absolutely) convergent. Similarly, for sample paths $y(s)$ of a stochastic process $Y_{T}$ the integral $\int_{[0, t]} d y(s)$ does not generally exist when considered as a Lebesgue-Stieltjes integral. But it exists for all sample paths $y_{T}$, with value $y(t)-y(0)$, when considered as a Stieltjes-complete integral.

There is no analytical cost or disadvantage in relinquishing the Lebesgue construction in favor of the -complete method. This is because the important theorems of Lebesgue integration, such as monotone and dominated convergence, are also valid for the -complete approach. Furthermore, there are other convergence theorems of a similar kind, specifically designed to deal with highly oscillatory functions such as those which occur in the theory of stochastic 
processes but which are beyond the scope of the Lebesgue method. See [13] for details of these.

However, stochastic integration includes novelties and challenges which have not yet been addressed in this essay.

For Brownian motion processes $X_{T}$, one of the most important stochastic integrals is $\int_{0}^{t} d X_{s}^{2}=t$. The corresponding integral for a sample path $x(s)(0 \leq s \leq t)$ is " $\int_{0}^{t}(d x(s))^{2}$ ". But this expression does not have the familiar form of a Stieltjes-type integral: $\int_{a}^{b} f(s) d g$, which, when $g$ is the identity function, reduces to the even more familiar $\int_{a}^{b} f(s) d s$.

In Riemann sum approximation we are dealing with expressions $\sum(x(I))^{2}$, where, for $\left.\left.I=\right] u, v\right], x(I)=x(v)-x(u)$. But traditionally, while a Riemann sum for a Stieltjes integral involves terms $f(s) x(I)$ with integrator function $x(I)$ (in which $f(s)$ can be identically 1 ), we do not usually expect to see integrators such as $(x(I))^{2}$ or $d X_{s}^{2}$.

Another important stochastic integral in Brownian motion theory is

$$
\int_{0}^{t} X_{s} d X_{s}=\frac{1}{2} X_{t}^{2}-\frac{1}{2} t
$$

For a sample path $x(s)$ of Brownian motion, this involves $\int_{0}^{t} x(s) d x(s)$, or, in Riemann sum terms, $\sum x(s) x(I)$. The latter, as it stands, is a finite sum of terms $x(s)(x(v)-x(u))$ where $I=] u, v]$ and $u \leq s \leq v$. And if we are using the Stieltjes-complete approach as described above, then we might suppose that each $s$ in the Riemann sum is the special point used in partitions which are constrained by a gauge $\delta(s)$

$$
s-\delta(s)<u \leq s \leq v<s+\delta(s) .
$$

But in fact this is not what is required in the stochastic integral $\int_{0}^{t} X_{s} d X_{s}$. In Riemann sum format, what is required is

$$
\sum x(u) x(I), \quad \text { or } \quad \sum x(u)(x(v)-x(u)),
$$

where the first factor $x(u)$ in the integrand is a point function evaluated at the left hand end-point $u$ of the interval $I=] u, v]$.

Sometimes the form $\sum x(w)(x(v)-x(u))$ is used, with $w=u+$ $\frac{1}{2}(v-u)$.

In a way, integrands of form $x(I)^{2}, x(u) x(I)$, or $x(w) x(I)$, are an unexpected innovation. Their value is calculated from the numbers 
$u$ and $v$ which specify the interval $I$. So they can be thought of as functions $h(I)$ of intervals $I$.

But these functions are not additive ${ }^{7}$ on intervals. In that regard they are unlike the integrators $|I|$ and $x(I)$ which are themselves functions of $I$ but are finitely additive on intervals, in the sense that, if $J=I_{1} \cup \ldots \cup I_{n}$ is an interval, then

$$
|J|=\sum_{j=1}^{n}\left|I_{j}\right|, \quad x(J)=\sum_{j=1}^{n} x\left(I_{j}\right) .
$$

Broadly speaking, integration is a summation process in which the summed terms involve functions of intervals. Up to this point in this essay, the only integrands to be considered included a factor which was an additive function of intervals $I$, such as the length function $|I|$ or the Stieltjes-type functions $g(I)$ or $x(I)$. But there is nothing inherent in the definition of -complete integrals that requires any $I$-dependent factor in the integrand to be additive.

With this in mind, consider again the definition of the -complete integral on an interval $[a, b]$.

Firstly, a gauge is a function $\delta(s)>0, a \leq s \leq b$. Given $s$, an interval $I=] u, v]$ for which $s$ is either an end-point or an interior point, is $\delta(s)$-fine if $s-u<\delta(s)$ and $v-s<\delta(s)$. A finite collection $\mathcal{D}=\left\{\left(s_{1}, I_{1}\right), \ldots,\left(s_{n}, I_{n}\right)\right\}$ is a division of $[a, b]$ if each $s_{j}$ is either an interior point or end-point of $I_{j}$ and the intervals $I_{j}$ form a partition of $[a, b]$. Given a gauge $\delta$, a division $\mathcal{D}$ is $\delta$-fine if each $\left(s_{j}, I_{j}\right) \in \mathcal{D}$ is $\delta$-fine.

Now suppose $h$ is a function of elements $(s, I)$. Examples include: $h(s, I)=h_{1}(I)=|I|, \quad h(s, I)=h_{2}(s)=s, \quad h_{3}(s, I)=$ $s^{2}|I|, \quad h_{4}(I)=|I|^{2}$. Given a division $\mathcal{D}=\{(s, I)\}$ of $[a, b]$ whose intervals $I$ form a partition $\mathcal{P}$, the corresponding Riemann sum is

$$
(\mathcal{D}) \sum h(s, I),=\sum\{h(s, I): I \in \mathcal{P}\} .
$$

Definition 9.1. A function $h(s, I)$ is integrable on $[a, b]$, with integral $\int_{[a, b]} h(s, I)=\alpha$, if, given $\varepsilon>0$. there exists a gauge $\delta(s)>0$ so that, for each $\delta$-fine division $\mathcal{D}$ of $[a, b]$,

$$
\left|\alpha-(\mathcal{D}) \sum h(s, I)\right|<\varepsilon .
$$

\footnotetext{
${ }^{7}$ If $h(I)$ were finitely additive on intervals $I$ it could be used to define a point function $h(s):=h([0, s])$, and vice versa. Integrals with respect to finitely additive integrators are therefore representable as Stieltjes-type integrals, and vice versa.
} 
Applying this definition to the examples, $h_{1}$ is integrable with integral $b-a, h_{2}$ is not integrable, $h_{3}$ is integrable with integral $\frac{1}{3}\left(b^{3}-a^{3}\right)$, and $h_{4}$ is integrable with integral 0 . If $h(s, I)=h_{5}(I)=$ $u^{2}|I|$ where, for each $I, u$ is the left hand end-point of $I$, then it is not too hard to show that $h_{5}$ is integrable with integral $\frac{1}{3}\left(b^{3}-a^{3}\right)$.

Actually, it is the traditional custom and practice in this subject to only consider integrands $h(s, I)=f(s) p(I)$ where the integrator function $p(I)$ is a measure function or, at least, finitely additive on intervals $I$; and where the evaluation point $s$ of the point function integrand $f(s)$ is the point $s$ of $(s, I)$ for each $(s, I) \in \mathcal{D}$. When $p(I)=|I|$, this convention is needed in order to prove the Fundamental Theorem of Calculus. 8 .

But, while the Fundamental Theorem of Calculus is important in subjects such as differential equations, it hardly figures at all in some other branches of mathematics such as probability theory or stochastic processes. And we have seen that stochastic integration often requires point integrands $f(s)$ to be evaluated, not at the points $s$ of $(s, I) \in \mathcal{D}$, but at the left hand end-points of the partitioning intervals $I$.

So, with $I=] u, v], f(u)$ is, in fact, an integrand function which depends, not on points $s$ but on intervals ] $u, v]$.

These are a few of the "unexpected innovations" to be encountered in stochastic integration, giving it a somewhat alien and counterintuitive feel to anyone versed in the traditional methods of calculus. Indeed, these are further examples of probability and analysis losing contact with each other.

For instance, the stochastic integral $\int_{0}^{t} X d X$ is given the value $\frac{1}{2} X(t)^{2}-\frac{1}{2} t$ when the process $X(s)$ (with $X(0)=0$ ) is a Brownian motion. Introductory treatments of this problem sometimes contrast the expression $\int_{0}^{t} X d X$ with the elementary calculus integral $\int x d x$ whose indefinite integral is $\frac{1}{2} x^{2}$, in which the use of symbols $X$ and $x$ can, in the mind of an inexperienced reader, set up an inappropriate and misleading analogy.

In terms of sample paths, the stochastic integral $\int_{0}^{t} X(s) d X(s)$ has representative sample form $\int_{0}^{t} x(s) d x(s)$ which is a Stieltjes-type integral with integrator function $x(I)=x(v)-x(u)$, formed from a typically "zig-zag" Brownian path $x(s), 0<s \leq t$, with $x(0)=0$.

\footnotetext{
${ }^{8}$ The Fundamental Theorem of Calculus states that if $F^{\prime}(s)=f(s)$ then $f(s)$ is integrable on $[a, b]$ with definite integral equal to $F(b)-F(a)$
} 
Then the notation for the contrasting elementary calculus integral is not $\int x d x$, but $\int s d s$, with value $\frac{1}{2} s^{2}$. Putting the latter in Stieltjes terms, $\int s d s$ is the Stieltjes integral $\int_{0}^{t} x(s) d x(s)$ where the sample path path or function $x$ is the identity function $x(s)=s, 0 \leq s \leq t$.

Clearly a Stieltjes integral involving a "typical" Brownian path $x(s)$ (which though continuous is, typically, nowhere differentiable) is a very different beast from a Stieltjes integral involving the straight line path $x(s)=s$. So in reality it is not surprising that there is a very big difference between the two integrals

$$
\int X(s) d X(s)=\frac{1}{2} X(t)^{2}-\frac{1}{2} t, \quad \text { and } \quad \int s d s=\frac{1}{2} s^{2} .
$$

The first integral typically involves Stieltjes integrals using very complicated and difficult Brownian paths $x(s)$. It should be distinguished sharply from the more familiar and simpler Stieltjes integrals in which, for instance, the point function component of the integrand is a continuous function, and the integrator or interval function is formed from increments of a monotone increasing or bounded variation function.

It is easy to overlook this distinction. Example 60 of [13] illustrates the potential pitfall. In this Example, $X_{T}$ is an arbitrary stochastic process and, with a fixed partition of $T=] 0, t], 0=\tau_{0}<\tau_{1}<\cdots<$ $\tau_{m}=t$, the function $\sigma(s)$ is constant for $\tau_{j-1}<s \leq \tau_{j}$. Example 60 claims, in effect, that the stochastic integral $\int_{\tau_{j-1}}^{\tau_{j}} \sigma(s) d X_{s}$ exists for each $j$ in the same way that, for constant $\beta, \int_{\tau_{j-1}}^{\tau_{j}} \beta d X_{s}$ exists and equals $\beta\left(X\left(\tau_{j}\right)-X\left(\tau_{j-1}\right)\right)$.

But Example 8.3 above shows that this claim is false. As a step function, $\sigma\left(\tau_{j-1}\right)$ is not generally equal to the constant $\beta=\sigma(s)$ when $s>\tau_{j-1}$. So if the sample path $x(s)$ is the Dirichlet function $D(s)$, the Stieltjes integral $\int_{\tau_{j-1}}^{\tau_{j}} \sigma(s) d x(s)$ does not exist, and the claim in Example 60 is invalid.

However, if $X_{T}$ is a Brownian motion process, then each of the significant sample paths $x(s)$ satisfies a condition of uniform continuity. In that case Example 60 is valid. But it requires some proof, similar to the proof of Theorem 229 on the succeeding page.

So what is truly surprising in (7) is, not that the two integrals give very different results, but that any convergence at all can be found for the first integral. 
Why is this so? This essay has avoided giving any precise meaning to expressions such as $\int_{0}^{t} X d X$ - or even to a random variable $X_{s}$. But the meaning of the random variable $\int_{0}^{t} X_{s} d X_{s}$ is somehow representative of a Stieltjes-type integral which can be formulated for every sample path $\{x(s): 0<s \leq t\}$. These sample paths may consist of joined-up straight line segments (as in the archetypical jagged-line Brownian motion diagram), or smooth paths, or everywhere discontinuous paths (like the Dirichlet function). Thus any claim that all of the separate and individual Stieltjes integrals $\int_{0}^{t} x(s) d x(s)$ of the class of such sample paths $x$ - a very large class indeed-have integral values $\frac{1}{2} x(t)^{2}-\frac{1}{2} t$ must be somehow challenging and dubious.

The integrals $\int_{0}^{t} d X(s)=X(t), \int_{0}^{t} d x(s)=x(t)$, show that each member of a large class of Stieltjes integrals can indeed yield a common, single, simple result. Our discussion of the Riemann sum calculation of these integrals illustrates how this happens: regardless of the values of $x(s)$ for $s<t$, adding up increments ensures that all values $x(s)$ cancel out, except the terminal value $x(t)$.

Thus, if $f(s)$ takes constant value $\beta$ for $0 \leq s \leq t$, then, for every sample path $x(s)$, the Riemann-Stieltjes (and Stieltjes-complete) integral $\int_{0}^{t} f(s) d x(s)$ exists, and $\int_{0}^{t} f(s) d x(s)=\beta x(t)$ (or $\beta(x(t)-$ $x(0))$ if $x(0) \neq 0$. This is the basis of the claim that the stochastic integral $\int_{0}^{t} f(s) d X(s)$ exists, and is the random variable $\beta X(t)$.

However, Example 8.3 demonstrates that caution must be exercised in pursuing further the logic of Riemann sum cancellation. Because if the sample path $x(s)$ is the function $D(s)$ of Example 8.3, the expression $f(s) D(I)$ is not integrable on $[0, t]$, in either the Riemann-Stieltjes sense or the Stieltjes-complete sense, even when $f(s)$ is a step function (non-constant).

It is indeed possible to take the Riemann sum cancellation idea further. Theorem 229 of [13] shows how this can be done.

But many important stochastic integrands are not actually integrable in the basic sense of the Definition 9.1. If various sample paths $x(s)$ are experimented with in the integral $\int_{0}^{t} d X_{s}^{2}$, many different results will be found. So what is the meaning of the result $\int_{0}^{t} d X_{s}^{2}=t ?$

While, for different sample paths $x, \int_{0}^{t} d x_{s}^{2}$ is not generally convergent to any definite value, there is a weak sense of convergence of the 
integral which makes " $\int_{0}^{t} d X_{s}^{2}=t$ " meaningful. Most importantly in this case, the weak limit $t$ is a fixed quantity rather than a random or unpredictable quantity such as $x(t)$. But this question goes beyond the scope of the present essay, whose aim is to explore some of the basic concepts of this subject, and hopefully to illuminate them a little. A more extensive exploration is presented in [13].

\section{REFERENCES}

[1] Bartle, R.G., Return to the Riemann integral, American Mathematical Monthly 103(8) (1980), 625-632.

http://mathdl .maa.org/images/upload_library/22/Ford/Bartle625632.pdf

[2] Bullen, P.S., Nonabsolute integration in the twentieth century, American Mathematical Society Special Session on Nonabsolute Integration, Toronto, 23-24 September, 2000, www . emis.de/proceedings/Toronto2000/papers/bullen.pdf

[3] Chung, K.L., and Williams, R.J., Introduction to Stochastic Integration, Birkhäuser, Boston, 1990.

[4] Itô, K., and McKean, H.P., Diffusion Processes and their Sample Paths, Academic Press, New York, 1965.

[5] Jarrow, R., and Protter, P., A short history of stochastic integration and mathematical finance: the early years, 1880-1970, http://people.orie.cornell.edu/ protter/WebPapers/ historypaper7.pdf

[6] Henstock, R., The efficiency of convergence factors for functions of a continuous real variable, Journal of the London Mathematical Society 30 (1955), $273-286$.

[7] Karatzas, I., and Shreve, S. E., Brownian Motion and Stochastic Calculus, Springer-Verlag, New York, 1991.

[8] Kolmogorov, A.N., Grundbegriffe der Wahrscheinlichkeitreichnung, Ergebnisse der Mathematik, Springer, Berlin, 1933 (Foundations of the Theory of Probability, Chelsea Publishing Company, New York, 1950).

[9] McKean, H.P., Stochastic Integrals, Academic Press, New York, 1969.

[10] McShane, E.J., A Riemann Type Integral that Includes Lebesgue-Stieltjes, Bochner and Stochastic Integrals, Memoirs of the American Mathematical Society No. 88, Providence, 1969.

[11] McShane, E.J., A unified theory of integration, American Mathematical Monthly 80 (1973), 349-359.

[12] McShane, E.J., Stochastic Calculus and Stochastic Models, Academic Press, New York, 1974.

[13] Muldowney, P., A Modern Theory of Random Variation, with Applications in Stochastic Calculus, Financial Mathematics, and Feynman Integration, Wiley, New York, 2012.

[14] Muldowney, P., A Riemann approach to random variation, Mathematica Bohemica 131(2) (2006), 167-188. 
[15] Muldowney, P., Henstock on random variation, Scientiae Mathematicae Japonicae, No. 247 67(1) (2008), 51-69.

[16] Øksendal, B., Stochastic Differential Equations, Springer-Verlag, Berlin, 1985.

Pat Muldowney served as lecturer at the Magee Business School of the University of Ulster for over twenty years. Dr. Muldowney's books include A General Theory of Integration in Function Spaces, Pitman, 1987 and A Modern Theory of Random Variation, with Applications in Stochastic Calculus, Financial Mathematics, and Feynman Integration, Wiley, 2012.

Lisselty Dunmore East Co. Waterford.

E-mail address: pat.muldowney@googlemail.com 